\title{
Morphological Segmentation of the Spleen From Abdominal CT Images
}

\author{
Belgherbi. Aicha \\ Biomedical engineering laboratory, Electrical and Electronics engineering Department \\ Tlemcen University, 13000, Algeria. \\ E-mail: mmebelladgham@yahoo.fr \\ Bessaid. Abdelhafid \\ Biomedical engineering laboratory, Electrical and Electronics engineering Department \\ Tlemcen University, 13000, Algeria \\ E-mail: a_bessaid@mail.univ-tlemcen.dz
}

\begin{abstract}
Organ segmentation is an important step in various medical image applications. Accurate spleen segmentation in abdominal CT images is one of the most important steps for computer aided spleen pathology diagnosis. In this paper, we have proposed a new semiautomatic algorithm for spleen area extraction in abdominal CT images. The algorithm contains several stages. A spleen segmentation method is based on watershed approach. The first, we seek to determine the region of interest by applying the morphological filters such as the geodesic reconstruction to extract the spleen. Secondly, a pre-processing method is employed. In this step, we propose a method for improving the image gradient by applying the spatial filters followed by the morphological filters. Thereafter we proceed to the spleen segmentation by the watershed transform controlled by markers. The new segmentation technique has been evaluated on different CT images, by comparing the semi-automatically detected spleen contour to the spleen boundaries manually traced by an expert. The experimental results are described in the last part in this work. The automated method provides a sensitivity of $95 \%$ with specificity of $99 \%$ and performs better than other related methods.
\end{abstract}

Index Terms- CT images, spleen segmentation, anisotropic diffusion filter, morphological filters, the watershed

\section{INTRODUCTION}

Many practical applications in medical image processing require robust and valid image segmentation for accurate analysis of anatomical structures.

Image Segmentation is the most fundamental technique for image analysis. Therefore, designing and developing a computer-aided diagnosis (CAD) tools for spleen is necessary to increase the productivity of radiologists who interpret and diagnosis hundreds of CT images every day. With increasing emphasis on medical image segmentation, many techniques have been proposed for this purpose.

Paola Campadelli et al 2008 used fast marching Technique' for spleen segmentation [1]. Alireza Behrad, Hassan Masoumi 2010 propose a new automatic algorithm for spleen segmentation via MRI image [2]. Either of them has advantages and disadvantages in terms of applicability, suitability, performance, and computational cost. In this work, we are interested to the watershed approach for the spleen segmentation. The Watershed segmentation technique has been widely used in medical image segmentation.

Advantages of the watershed transform include the fact that it is a fast, simple and intuitive method. More significantly, it is able to produce a complete division of the image in separated regions even if the contrast is poor [3]. The objective of our work is the spleen segmentation. To address above problems, we present a semi-automatic spleen segmentation algorithm in abdominal CT images using the mathematical morphology in particular the watershed approach. Despite its advantages, the watershed segmentation technique has some drawback which includes over-segmentation. A common method is the use of region markers. In this paper, morphological reconstruction is applied to remove salient parts which hinder proper segmentation. In addition, we proposed an algorithm used for improving the image gradient in order to solve over-segmentation problem. In these tasks, we employed the anisotropic diffusion filter used to smooth the original images. After that, we used the morphological filter for improving the image gradient toward to ensure a satisfying image segmentation result.

The paper is organized as follows. The watershed method spleen segmentation process is described in section II. Our algorithm of segmentation by the watershed will be detailed in section III. The experimental results are presented in section IV. Finally the paper concludes in section $\mathrm{V}$. 


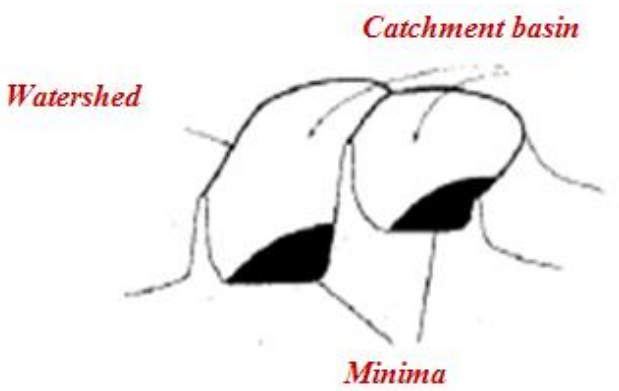

Figure 1. Watershed Segmentation simplified in 2 dimensions

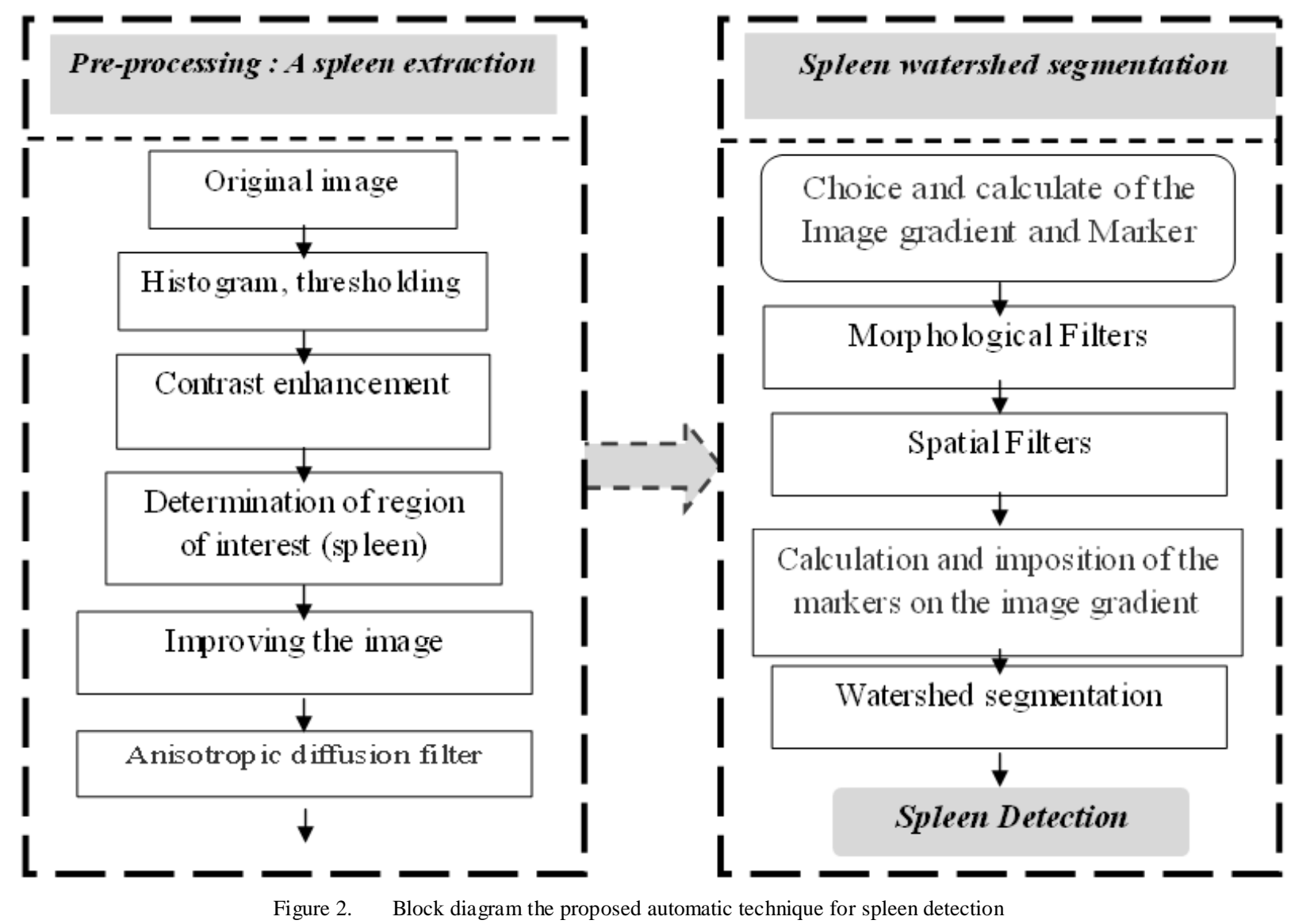

\section{WATHERSHED TRANSFORM}

The Watershed is powerful region based image segmentation algorithm [4]. Since its introduction Watershed was introduced by Beucher and Lantuejoul [5], it has been widely studied and achieved encouraging results in image segmentation [6].

Its basic idea is to consider image as topography topology of geodesy Fig.1, the pixel gray value of each point of image stands for the altitude of that point, every local minimum value and its impact region are known as the collection basin and the borders of the basin form the watershed [7].

Intuitively, the watershed of a function is composed of the locations from which a drop of water could flow towards different minima [8]. The immersion process is simulated from the heights of local minima. The water rises in each basin and when two basins meet, a watershed is created between them [9].

\section{IMPLEMENTATION OF THE PROPOSED METHOD BASED ON WATERSHED SEGMENTATION}

The block diagram of the proposed method is shown in Fig.2. Each block will be explained in the following sections

\section{A. Pre-processing}

Most of abdominal CT images are noisy and the edges of objects are not clear enough in these images. To handle this problem, we applied preprocessing stage to the input image before applying the main segmentation stage.

In order to reduce over-segmentation, several strategies may be considered: defining a robust gradient, or setting the predefined number of regions with markers which define the initial catchment basins. 
The aim of this stage is to reduce the noisy artifacts while preserving, as much as possible, the original image structures and improving the gradient to facilitate the spleen segmentation

1)Morphological reconstruction: In this paper, the salient parts of input images are removed by using morphological reconstruction, which uses marker and mask images. One of the most important operations in morphological image processing is reconstruction from markers. Morphological reconstruction is a nonlinear filter based on mathematic morphology. Comparing to other types of filters, morphological reconstruction can

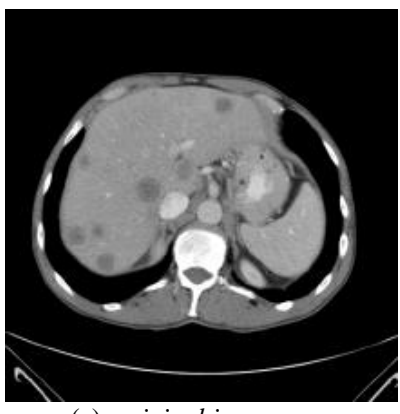

(a): original image

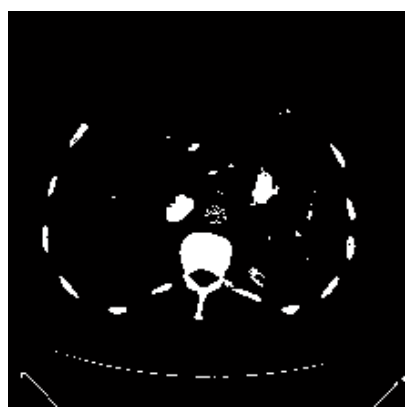

(b): thresholding of (a) keep the information of object contours when filtering the image [10]. In morphological reconstruction, the peaks in the marker image "spread out," or dilate. Each successive dilation is constrained to lie underneath the mask. When further dilation ceases to change the image, processing stops. The final dilation is the reconstructed image. Eq. (1) shows the reconstruction procedure, in which the initial marker image obtained from erosion of the original image and $\mathrm{g}$ is the mask image. $\mathrm{B}$ is a $\mathrm{SE}$ (structuring element), and $\bigoplus$ is the dilation operator [11]

$$
h_{k+1}=\min \left(\left(h_{k} \oplus B\right), g\right)
$$

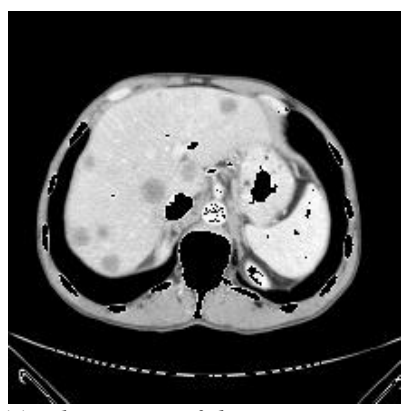

(c): elimination of the contracts zone

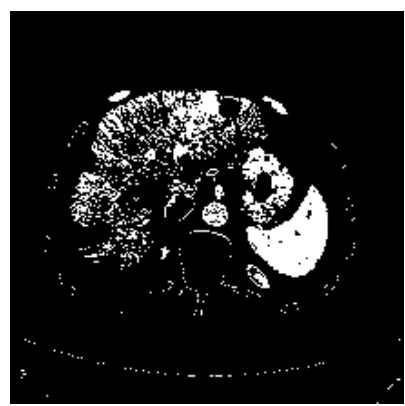

(d): threshold of $(c)$

Figure 3. Extraction of the region of interest ROI.

Spleen-ribs and the kidney separation. The first stage consists the spleen extracting. This stage is carried by a morphological reconstruction. But the spleen is connected to the ribs and kidney Fig.3.a, which will distort the final result of the extraction of ROI. In the first step, we remove the contrasted zones.

The contrast zones are extracted by using a morphological filter around a dilation geodesic reconstruction Fig.3.d. The marker image is obtained by thresholding the original image Fig.3.d. To ensure an excellent extraction of the region of interest, it is necessary to make a judicious choice of the threshold.

Then, the neighbor organs are separated using erosion operating by a structuring element with small value. This operation will be followed by a filtering according to surface criterion to eliminate the pixels insulate. The image marker will be iteratively transformed by geodetic dilations. Finally the geodesic reconstruction by dilation permits to extract the region which will be eliminated The contrast zones are removed by a simple subtraction between the original image and the image resulting from the morphological reconstruction Fig.3.c.

The spleen detection: To detect semi-automatically the contour delimiting the spleen, the developed algorithm analyzes initially the intensity distribution either of the spleen and the adjacent organs. To extract the spleen only, our algorithm used a set of markers. Thresholding operation is applied to the image without the contrasted zones Fig.3.d. We emphasize that the choice of the threshold is very important when the spleen is needed to be marked. The application of the morphological reconstruction permit to extract the spleen
Fig.4.a . After, we apply a space filtering by anisotropic diffusion Fig.4.b.

Most of abdominal CT images are noisy and the edges of objects are not clear enough in these images. To handle this problem, we applied preprocessing stage to the input image before applying the main segmentation stage

2) Anisotropic diffusion filter: The ADF is a popular filtering process aiming to eliminate the noise from an image while preserving contours [12].

CT data are smoothed using anisotropic diffusion to enhance the homogeneity of abdominal objects and ensure boundary preservation. We employ the classic Perona-Malik anisotropy model [13]. During the diffusion process, smoother versions of an image $I$ are computed iteratively

$$
\left\{\begin{array}{c}
\frac{\partial I(x, y, t)}{\partial t}=\operatorname{div}(g(\|\nabla I(x, y, t)\|) \cdot \nabla I(x, y, t)) \\
I(x, y, 0)=I_{0}(x, y) \\
\left.\frac{\partial I}{\partial n}\right|_{\partial \Omega}=0
\end{array}\right.
$$

Where $d i v$ is a divergence operator and $\nabla$ is a gradient operator. $I$ in our case is the 2D of CT image, t: time, Two different diffusion functions have been suggested:

$g(I)=\exp \left(-\left(\frac{|\nabla I|^{2}}{K}\right)\right)$ 


$$
g(I)=\frac{1}{1+\left(\frac{|\nabla I|}{K}\right)^{2}}
$$

The coefficient $K$ is called coefficient of diffusion. This filter decreases the over-segmentation resulting from the application of the Watershed method Fig.4.b. This type of filtering is interesting especially when the image is noised

\section{B. Calculation of the gradient}

The watershed algorithm is usually carried out in the gradient image. There are three different combinations:

- The Beucher gradient which is the arithmetic difference between the dilated and eroded image:

$$
\operatorname{Grad}(f)=\delta_{B}(f)-\varepsilon_{B}(f)
$$

- The intern gradient which corresponds to the arithmetic difference between the original image and the eroded image,

$$
\operatorname{Grad}^{-}(f)=f-\varepsilon_{B}(f)
$$

- The extern gradient which represents the arithmetic difference between the dilated image and the original image,

$\operatorname{Grad}^{+}(f)=\delta_{B}(f)-f$

In our case we have objects (spleen) clearer than the bottom so, the internal gradient generates less thick contours compared to the two other gradients, which is desirable, in addition, the internal gradient delimits the inside of the object, as a result the use of the internal gradient is recommended in our application [14].

Direct application of the watershed transform to a gradient image usually leads to over-segmentation due to noise and other local irregularities of the gradient.

To prevent any over-segmentation error (e.g. vessels and parts of neighboring organs...), the morphological gradient modification method can be employed. We smooth the ROI surface by another morphological filter such as opening by reconstruction Fig.4.c. After, we calculate the second spatial filter. Any one of the gradient operators like Sobel, Prewitt or Gaussian derivative can be used. Since the noise level is efficiently reduced by filtering, these operators perform well on filtered images [8]

1) Opening by reconstruction [15]: The opening by reconstruction of the image $f$ is defined as the reconstruction by dilation of $\mathrm{f}$ by erosion of $\varepsilon(f)$

$$
\gamma_{R}(f)=\operatorname{Re} c_{\oplus}(\varepsilon(f), f)
$$

Where:
$R e c_{\oplus}$ define the reconstruction by dilation of $f, \varepsilon(f)$ the erosion of image $f$

$\operatorname{Rec}_{\oplus f}(g)(x, y)=\quad v_{\geq 1}^{\vee} \delta_{f}^{(n)}(g)(x, y)$

With the grayscale dilation of size $n \geq 0$ is given by:

$\underbrace{\delta_{f}^{(n)}(g)(x, y)=\delta_{f}^{(1)} \circ \delta_{f}^{(2)} \circ \ldots . \delta_{f}^{(n)}(g)(x, y)}_{\text {ntimes }}$

Openings-by-reconstructions are anti-extensive removing bright image details [16].

In medical imagery, this kind filter is interesting, it permit to remove vessels and small regions and detaches protrusion wrongly included into the spleen volume Fig.4.c.

The following stage permit to calculate the internal gradient:

1) Smoothing the CT images by an anisotropic diffusion filter Fig.4.b.

2) Application of the morphological filter 'opening by reconstruction' in order to eliminate the vessel and contrasts small objects Fig.4.c

3) Application of the second spatial filter

4) Image gradient Fig.4.g

\section{Calculation of the marker}

The first step consists to calculate the internal and external markers. The concept of markers can be used to solve the over-segmentation problem whose goal is to detect the presence of homogeneous regions from the image by a set of morphological simplifications. The marker involves production of markers by identifying the interior and exterior of the objects to be segmented. We relate hole filling Fig.4.d. A dilation operation is applied to this result with a disc-structuring element. The external marker is calculated by the internal gradient Fig.4.e. For the internal marker, we used the distance function Fig.4.f. Using this technique, it is possible to apply the watershed algorithm controlled by marker on the gradient [17], Fig.4.h, Fig.5.

\section{Watershed algorithm relative to the marker}

The Watershed segmentation algorithm applied directly to the gradient image can cause oversegmentation due to serious noise patches or other image irregularities [18]. Sometimes, it is desirable to use a set of markers instead of the minima of the map to fix the number of regions. To do this, one has to proceed to a morphological reconstruction of the map before computing the watershed [19].

\section{RESULT AND DISCUSCION}

We propose a method of the spleen segmentation by the watershed algorithm. Our algorithm was implemented on Matlab environment. The CT images collected from radiological department of Hospital of 
Tlemcen University of Algeria are used to demonstrate the performance of the proposed segmentation method. To evaluate the performance of our approach, we compared the automatic segmentation with the manual segmentation traced by expert. We evaluated our algorithm on datasets by computing four parameters: sensitivity, specificity, accuracy, and error rate as shown in Table. I and defined in Equations (11) to (14):

$$
\text { sensitivity }=T p /(T p+F n)
$$

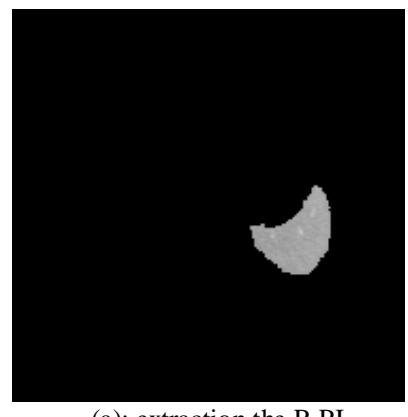

(a): extraction the R.PI

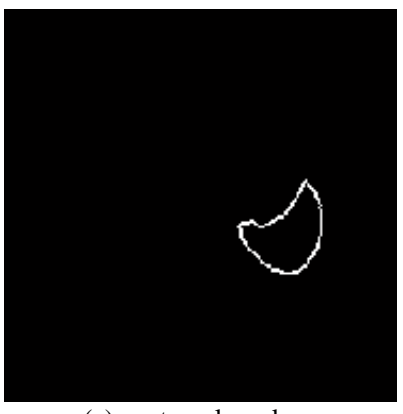

(e): external marker

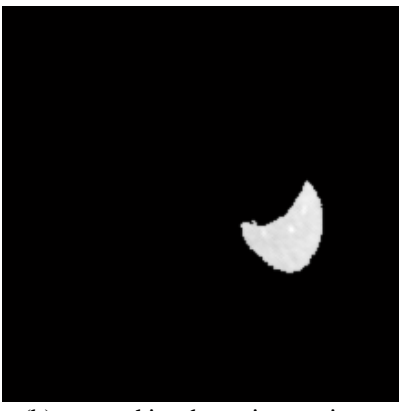

(b): smoothing by anisotropic diffusion filter

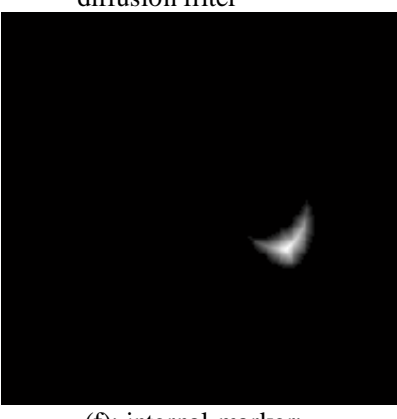

(f): internal marker: distance function

$$
\text { specifity }=\operatorname{Tn} /(\operatorname{Tn}+T p)
$$$$
\text { accuracy }=(T p+T n) /(T p+T n+F p+F n)
$$

$$
\text { Errorrate }=(F p+F n) /(T p+T n+F p+F n)
$$

\begin{tabular}{|c|c|c|c|c|}
\hline Dataset & Sensitivity & Specificity & accuracy & Error Rate \\
\hline 1 & 0.9282 & 0.9999 & 0.9972 & 0.0028 \\
\hline 2 & 0.9432 & 0.9999 & 0.9971 & 0.0029 \\
\hline 3 & 0.9352 & 0.9999 & 0.9986 & 0.0014 \\
\hline 4 & 0.9688 & 0.9998 & 0.9984 & 0.0016 \\
\hline 5 & 0.9355 & 0.9999 & 0.9355 & 0.0028 \\
\hline 6 & 0.9892 & 0.9997 & 0.9994 & $6.4038 \mathrm{e}-004$ \\
\hline 7 & 0.9669 & 0.9993 & 0.9976 & 0.0024 \\
\hline 8 & 0.9001 & 0.9997 & 0.9960 & 0.0040 \\
\hline 9 & 0.9270 & 0.9999 & 0.9979 & 0.0021 \\
\hline 10 & 0.9760 & 0.9985 & 0.9974 & 0.0026 \\
\hline 11 & 0.9338 & 0.9999 & 0.9972 & 0.0028 \\
\hline 12 & 0.9696 & 0.9998 & 0.9991 & $8.6038 \mathrm{e}-004$ \\
\hline 13 & 0.9454 & 0.9998 & 0.9973 & 0.0027 \\
\hline 14 & 0.9333 & 0.9997 & 0.9983 & 0.0017 \\
\hline 15 & 0.9507 & 0.9997 & 0.9980 & 0.0020 \\
\hline 16 & 0.9827 & 0.9975 & 0.9961 & 0.0039 \\
\hline 17 & 0.9396 & 0.9998 & 0.9973 & 0.0027 \\
\hline Average & 0.9502 & 0.9975 & 0.9935 & 0.0019 \\
\hline
\end{tabular}

Figure 4. Results of the liver segmentation by our method

TABLE I. EVALUATION OF THE RESULTS BASED ON OUR APPROACH 
With $T p$ : the number of truth positive; $T n$ : the number of truth negative, $F p$ : the number of the false positive and $F n$ is the number of the false negative.

The suggested technique gives promising results as indicate in Table. I. Sensitivity and specificity for semi-
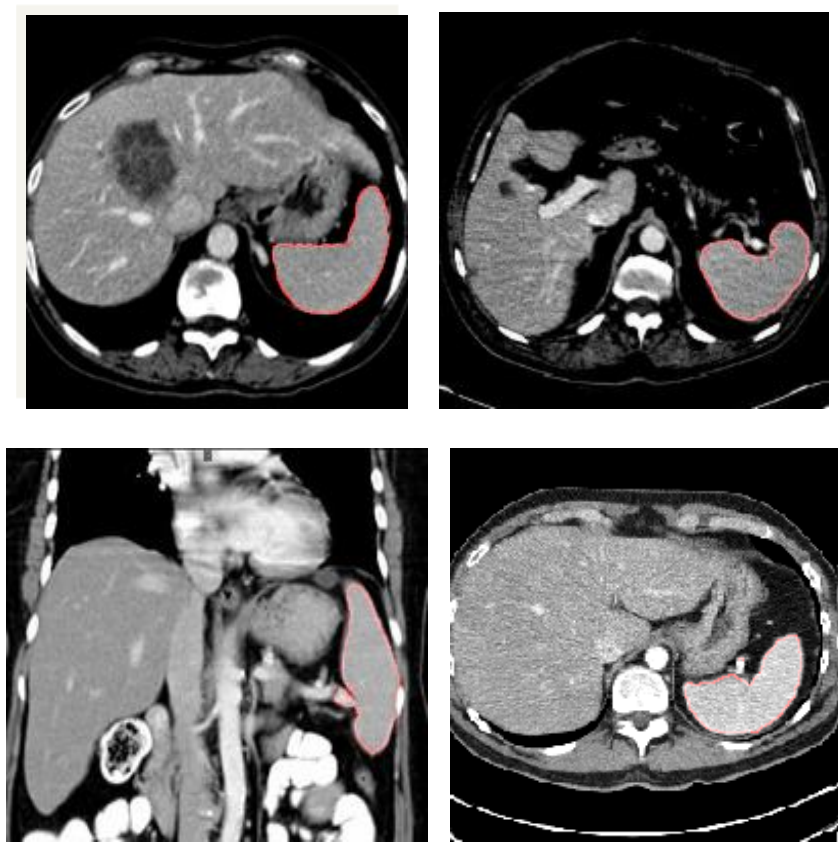

Figure 5. Spleen segmentation by our method for various images. automatic spleen segmentation are $95 \%$ and $99 \%$ respectively. As a result, the application of our algorithm for the detection of contours of the spleen gives attracting results.
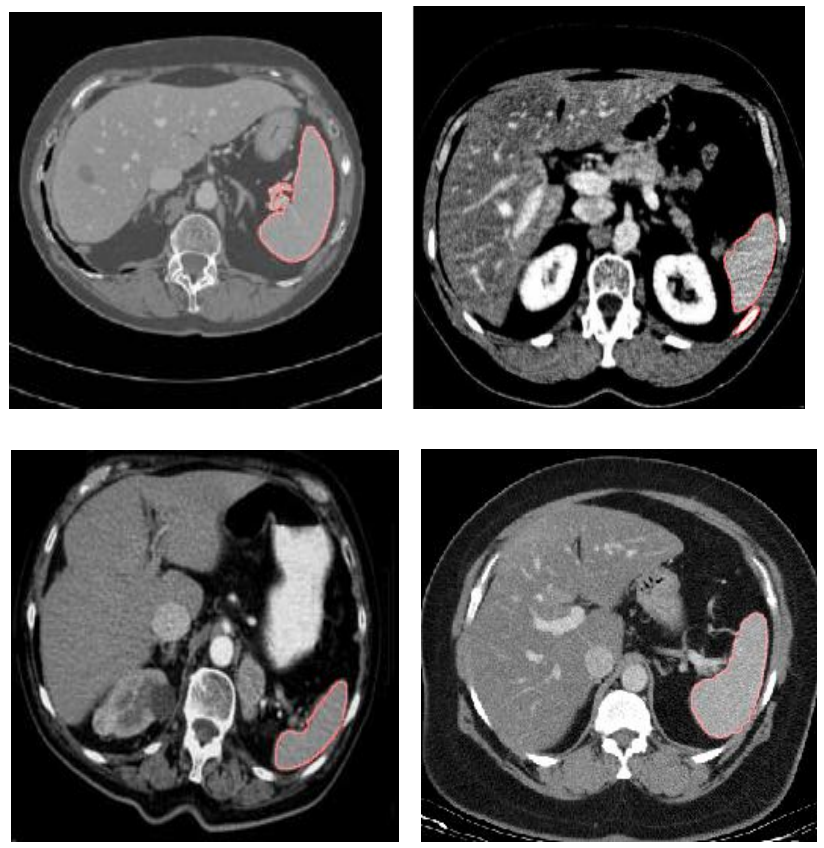

operation will be realized by morphological

TABLE II. THE AVERAGE RESULTS OF PREVIOUS WORKS THAT USED SAME METRIC

\begin{tabular}{|l|l|l|}
\hline Algorithm & $\begin{array}{l}\text { Paola Campadelli et } \\
\text { al 2010 }\end{array}$ & Proposed method \\
\hline Sensitivity & $94.4 \%$ & $95 \%$ \\
\hline
\end{tabular}

However, in some cases, such as datasets numbers 1 , 8 and 9 in Table.I the results are not satisfactory. These are generally due to the low contrast, neighboring organs such as muscles, the ribs and the kidney.

In the Table. II, we report the spleen segmentation results achieved by two algorithms. In [20] propose a fully automatic, gray-level based segmentation framework based on a multiplanar fast marching method; they stated sensitivity as $94.4 \%$. In our system achieves a mean sensitivity equal to $95 \%$. However as it is shown in Table II, the accuracy of the proposed algorithm is better than [20].

In the future work, we seek these problems and improve our approach for the spleen segmentation. Also the work can be extended in order to detect the hepatic lesions.

\section{CONCLUSION}

In this paper, we have proposed a semi-automatic segmentation algorithm of the spleen using mathematical morphology based on watershed approach. The semiautomatic method used consists to put two parameters manually. Our aim is the spleen segmentation but this organ connected to the ribs and kidney. To prevent this problem we seek to eliminate the contrast zones. This reconstruction where we used the thresholding of the original image. To ensure an excellent extraction of the region of interest, it is necessary to make a judicious choice of the threshold. This parameter change from the image to another.

The second parameter which will be set by the manipulator is the $h$ parameter of the morphological filter (opening by reconstruction) in order to calculate the gradient image.

The algorithm that we implemented is tested on 17 images TDM. We carried out initially the simplification of images by the use of the anisotropic diffusion filter followed by morphological filter and a morphological reconstruction. Besides, morphologic operation is applied to guarantee a fast and accurate spleen region. The final results are compared the automatic method with manually segmented image by the radiologist. The results of experiments show that the algorithm has a certain degree of adaptability and accuracy for the first step of the computer-aided diagnosis (CAD) and computer-aided surgery (CAS) systems.

\section{REFERENCES}

[1] Campadelli. P., E. Casiraghi, and S. Pratissoli, (2008). Fully Automatic Segmentation of Abdominal Organs from CT Images Using Fast Marching Methods," in Proceedings of the 2008 21st IEEE International Symposium on Computer-Based Medical Systems Volume 00, 2008, pp. 554-559

[2] Alireza Behrad, Hassan Masoumi, Automatic Spleen Segmentation in MRI Images using a Combined 
Neural Network and Recursive Watershed Transform, $10^{\text {th }}$ Symposition on Neural Network Application in , Electrical Engineering, NEURAL2010, 978-1-4244-8820-9/10/\$26.00 C2010 IEEE

[3] H.P. Ng1, S. Huang1, S.H. Ong2, 3,K.W.C. Foong4, P.S. Goh5, W.L. Nowinski1, Medical Image Segmentation Using Watershed Segmentation with Texture-Based Region Merging , 978-1-4244-18152/08/\$25.00 @2008 IEEE

[4] Eric Dahai Cheng, Subhash Challa and Rajib Chakravorty, Microscopic Cell Segmentation and Dead Cell Detection Based on CFSE and PI Images by Using Distance andWatershed Transforms, 978-0$7695-3866-2 / 09 \$ 26.00 \quad$ (C) 2009 IEEE DOI 10.1109/DICTA.2009.16

[5] Beucher S,Lantuejoul C, 1979: Use of watersheds in contour detection, Proceedings of International Workshop on Image Processing, Real Time Edge and Motion Detection/Estimation, Rennes, September 1979

[6] Yun Zhang, Xuezhi Feng, Xinghua Le, Segmentation on multispectral remote sensing image using watershed transformation 978-0-7695-3119$9 / 08 \$ 25.00 \quad$ C $\quad 2008$ IEEE DOI 10.1109/CISP.2008.365

[7] Qiao Sun, Wei Yang, Lina Yu, Research and Implementation of Watershed Segmentation Algorithm Based on CCD Infrared Images, 978-0$7695-4180-8 / 10 \quad \$ 26.00 \quad$ C 2010 IEEE DOI 10.1109/PCSPA.2010.127

[8] Camille Couprie, Leo Grady, Power watersheds: A new image segmentation framework extending graph cuts, random walker and optimal spanning forest, 2009 IEEE 12th International Conference on Computer Vision (ICCV) 978-1-4244-44199/09/\$25.00 @2009 IEEE

[9] Lahcen Moumoun, Mohamed El far, Mohamed, Chahhou, Taoufiq Gadi, Rachid Benslimane, Solving the 3D watershed over-segmentation problem using the generic adjacency graph, 978-1-4244-59988/10/\$26.00 @2010 IEEE

[10] Xiaoyan Zhang, Yong Shan, Wei Wei, Zijian Zhu, An Image Segmentation Method Based on Improved Watershed Algorithm, 978-0-7695-4270-6/10 \$26.00 (C) 2010 IEEE. DOI 10.1109/ICCIS.2010.69

[11] Myeong Yoo, Toan Nguyen Dinh, GueeSang Lee, Segmentation by Morphological Reconstruction and Non-Linear Diffusion, 0-7695-2983-6107\$25.00 0 2007 IEEE. DO1 10.1 109/CIT.2007.162

[12] Z. Peter V. Bousson, C. Bergot, F. Peyrin, A constrained region growing approach based on watershed for the segmentation of lowcontrast structures in bone micro-CT images, Pattern Recognition 41 (2008) 2358 - 2368, 00313203/\$30.00 _ 2008 Elsevier Ltd. All rights reserved doi:10.1016/j. patcog.2007.12.011

[13] Marius GeorgeLingurarua,* JianhuaYaoa, RabindraGautamb, JamesPetersonb, ZhixiLia, W., Renal tumor quantification and classification in contrast-enhanced abdominal CT MarstonLinehanb,
RonaldM.Summersa 0031-3203/\$ -seefrontmatter (C) 2008 doi:10.1016/j.patcog.2008.09.018

[14] Benoit Naegel, 'Abdominal organs segmentation by a topological and morphological creterian, thesis , Louis Pasteur university, Strasbourg, october 2004

[15] Heimann, T., Wolf, I., Meinzer, H.P., Active shape models for a fully automated $3 \mathrm{~d}$ segmentation of the liver - an evaluation on clinical data, In: Larsen, R., Nielsen, M., Sporring, J. (Eds.), MICCAI 4191 of LNCS. Springer-Verlag, pp. 41-48. 2006

[16] Michael H.F. Wilkinson, connected filtering by reconstruction: basis and new advances, institute of mathematics and computing science, university of Groningen, 978-1-4244-1764-3/08/\$25.00 (C2008 IEEE

[17] Cédric Allène, Jean-Yves Audibert, Michel Couprie, Renaud Keriven,' 'Some links between extremum spanning forests, watersheds and min-cuts', Image and Vision Computing 28 (2010) 1460-1471

[18] Nallaperumal Krishnan, Senior Member, IEEE, Krishnaveni. K, Member, IEEE, (2006). An efficient Multiscale Morphological Watershed Segmentation using Gradient and Marker Extraction ,Justin Varghese, 1-4244-0370-7/06/\$20.00 @2006 IEEE

[19] Allène Cédric, Audibert Jean-Yves , Couprie Michel, Keriven Renaud, (2010). Some links between extremum spanning forests, watersheds and min-cuts, Image and Vision Computing 28 (2010) 1460-1471

[20] Paola Campadelli, Elena Casiraghi *, Stell Pratissoli, A segmentation framework for abdominal organs from CT scans, Artificial Intelligence in Medicine 50 (2010) 3-11

Belgherbi. Aicha received the Diploma Engineering degree from the Tlemcen University of Technology in 2005. She interest in medical image processing. She had obtained its master degree diploma in biomedical image processing in 2009 from the Tlemcen University of Technology. From 2009 until 2012 she works as a researcher in Electrical and Electronics engineering Department of Tlemcen University. Currently, she is in third year Ph.D student in biomedical engineering at the University of Tlemcen -Algeria. Here research focuses on mathematical morphology application to the biomedical image processing. From 2010 until 2012, she was a member of national research project NRP. From 2010 until 2011, 2011-2012, she was a member of tutored system; she gives courses in biomedical signal processing and numerical method

Bessaid. Abdelhafid is Professor in Dept. of Electronic \& Electrical Engineering at University of Tlemcen. He was born in Tlemcen, Algeria. He received the dipl. El.-Ing. degree from the University of Sciences and Technology of Oran (USTO, Algeria); the Magiter degree and the PHD from the University of Sidi Bel Abbes (Algeria), respectively in 1981, 1991 and 2004. He Works, since 1996, in the field of Medical Imaging and Image Processing at University of Tlemcen, Algeria 